\title{
BMJ Open Implementation of the partograph in India's JSY cash transfer programme for facility births: a mixed methods study in Madhya Pradesh province
}

\author{
Sarika Chaturvedi, ${ }^{1,2}$ Sourabh Upadhyay, ${ }^{1}$ Ayesha De Costa, ${ }^{2}$ Joanna Raven ${ }^{3}$
}

To cite: Chaturvedi S, Upadhyay S, De Costa A, et al. Implementation of the partograph in India's JSY cash transfer programme for facility births: a mixed methods study in Madhya Pradesh province. BMJ Open 2015;5:e006211.

doi:10.1136/bmjopen-2014006211

- Prepublication history and additional material is available. To view please visit the journal (http://dx.doi.org/ 10.1136/bmjopen-2014006211).

Received 24 July 2014 Revised 23 January 2015 Accepted 24 January 2015

\section{CrossMark}

${ }^{1}$ Department of Public Health and Environment, R D Gardi Medical College, Ujjain, India ${ }^{2}$ Department of Public Health Sciences, Karolinska Institutet, Stockholm, Sweden

${ }^{3}$ Department of International Public Health, Liverpool School of Tropical Medicine, Liverpool, UK

Correspondence to Dr Sarika Chaturvedi; sarikabharat2005@gmail.com

\section{ABSTRACT}

Objectives: To study implementation of partograph use to monitor labour in facilities providing the JSY (Janani Suraksha Yojana) cash transfer programme for facility births in India by determining (1) adherence to partograph use, (2) staff abilities at partograph use and (3) staff responsiveness to the policy on partograph use. Design: A mixed methods study using Carroll's framework for implementation fidelity. Methods include (1) obstetric case record review, (2) a vignette-based survey among nurse midwives and (3) interviews with staff.

Setting: Routine use of the partograph is recommended to monitor progress of labour in most low-and middle-income countries (LMICs), including India, although currently available evidence in this regard is insufficient. This study was conducted in the context of the highly successful JSY programme in three districts of Madhya Pradesh province.

Participants: 73 different level JSY programme facilities participated in the record review, 233 nurse midwives at these facilities participated in the vignette survey and a total of 11 doctors and midwives participated in the interviews.

Results: The partograph was used in $6 \%$ of the 1466 records reviewed. The staff obtained a median score of 1.08 (maximum of 10) at competence in plotting a partograph. Three themes emerged from the qualitative data: (1) partographs are used rarely and retrospectively; (2) training does not support correct use of the partograph; and (3) partographs can be useful but are not feasible.

Conclusions: Implementation fidelity of partograph use in the JSY programme is low. Successful implementation of the partograph can result in improved quality of care in the JSY programme only if potential moderators to its adherence, such as training, supervision, staff 'buy in' and practice environment are addressed so that staff find a conducive practice environment in which to use the partograph and women find it beneficial to present early in labour.

\section{INTRODUCTION}

Skilled attendance at birth is central among strategies to reduce perinatal mortality and

\section{Strengths and limitations of this study}

- This is the first report of a detailed assessment of partograph use in facilities in a low-and middle-income country (LMIC) outside the African region.

- Use of an analytical framework to study implementation fidelity of partograph use increases relevance of the results to other LMICs struggling to promote partograph use and to reduce perinatal mortality.

- Use of mixed methods and multiple data sources provides a comprehensive exploration of issues with partograph use.

- The findings provide insights to consider when recommending for or against routine use of the partograph in resource constrained settings.

- This work offers an important contribution to the literature on quality of obstetric care in the context of a large cash transfer programme.

- Our results on adherence to partograph use are based on a record review. We were limited by the quality of clinical records and unable to determine the proportion of women who arrived late in labour.

- Our study is restricted to public facilities in Madhya Pradesh; hence caution is suggested when generalising our findings to public facilities in other provinces or to private facilities.

morbidity. Quality of care at childbirth is a critical determinant of outcomes of care. Coverage of skilled attendance at birth has received much attention especially since the articulation of the Millennium Development Goals (MDGs), however, the quality of obstetric care provided remains a neglected area, specifically in resource poor contexts. ${ }^{1}{ }^{2}$ The lack of appropriate and feasible indicators ${ }^{3}$ makes it difficult to meaningfully measure and compare quality of obstetric care across settings. The most commonly used measures today are the UN indicators for monitoring emergency obstetric care. ${ }^{4}$ These focus on 
the availability and density of facilities able to provide care for life-threatening obstetric complications as indicators of service provision, and of responsiveness of the

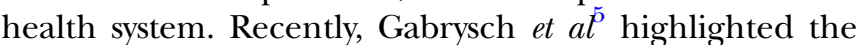
limitations of these popular indicators specifying that they do not reflect the quality of routine care for normal labour, which is equally important. They propose additional indicators for this purpose that include use of the partograph as routine for childbirth care. The World Health Organization has recommended, based on the findings of a large study in South East Asia, that the partograph be used in all labour wards. ${ }^{6}$ However, a recent Cochrane review ${ }^{7}$ identified that there was insufficient evidence to support the routine use of the partograph as standard part of labour management and care. The authors concluded that decisions regarding whether or not to use a partograph and which one to use should be locally determined. Nevertheless, many facilities in resource rich/poor settings currently use a partograph and have reported benefits in terms of ease of recording, provision of pictorial overview of progress, auditing of care, training of clinicians and transferring of care. ${ }^{8-10}$ However, reports from low-resource settings show low usage of the partograph and indicate challenges to its routine use, such as insufficient knowledge, non-availability of preprinted partographs and workload pressure. ${ }^{11}$ Other scholars have questioned the utility of its routine use, particularly in high-income settings, where competent individual clinical judgement is given more weight. ${ }^{12} 13$

India contributes to about a fourth of the global maternal death burden. ${ }^{14}$ Hence India's success at improving these outcomes significantly influences global improvements in maternal health. India has made remarkable progress in increasing the proportion of births occurring in health institutions-from $39 \%$ in 2007 to $73 \%$ in 2012. ${ }^{15}$ This was facilitated by the launch of the Janani Suraksha Yojana $(\mathrm{JSY})^{16}$ cash transfer programme in 2005 , which pays women to deliver in public institutions and in accredited private health facilities. The JSY aims to promote access to skilled attendance at birth by increasing facility births and so reduce perinatal mortality. With the JSY, the Indian Government also launched strategies to promote the use of the partograph to improve the quality of care during labour. Prominent among these is the 3-week residential skilled birth attendant ${ }^{17}$ (SBA) training where nursing staff from public sector facilities, who are already expected to be trained in partograph use during basic nursing education, are given skill-building opportunities. Since 2005, there have been other investments in maternal health services that complement the JSY programme, including easily accessible free emergency transportation for pregnant women. However, maternal mortality reduction in India has shown a secular trend since the beginning of the last decade. A number of scientific reports have failed to detect an effect of the JSY on reduction in maternal mortality ${ }^{18} 19$ despite its clear success with sharply rising institutional delivery proportions. This situation indicates possible deficiencies in quality of care provided under the programme that merit investigation. ${ }^{20} 21$

The use of the partograph has been recommended as an important indicator to monitor routine intrapartum care. We report on the use of the partograph as an indicator of intrapartum care in the JSY programme. Given that partograph use is actively promoted by the Indian government, and the required training and supplies are provided, questions pertaining to its implementation in the context of the JSY programme need to be answered. The degree to which an intervention is implemented as intended is termed as 'implementation fidelity', and its study is important to determine why and how interventions work, and possibilities of improved outcomes from its use. ${ }^{22}$ In this paper, we apply the framework for implementation fidelity proposed by Carroll et $a l^{23}$ The specific aspects we aimed to explore are adherence, ability and responsiveness to partograph use by answering the questions: 'What is the extent of use of the partograph during labour in JSY programme facilities (adherence)?' and 'Can it be used appropriately (ability) and do the nurse-midwives working in the programme 'buy in' to the policy of routine partograph use (responsiveness)?'.

This mixed methods study on implementation of partograph use has global relevance as it fills important gaps in knowledge specifically from low-income settings. Second, the JSY is the world's largest cash transfer programme; these results are of interest to those focusing on quality of provision of care in the context of cash transfer programmes for maternal mortality reduction.

\section{METHODS}

\section{Study framework}

In this study, we use the implementation framework developed by Carroll et al. ${ }^{23}$ This framework, unlike others, includes moderating factors that are likely to influence the degree of fidelity in implementation, and recognises the complex relationships between these factors. The strength of the framework lies in its ability to draw pragmatic solutions to improve outcomes from the intervention by consideration of the moderating factors and components essential to improve implementation.

In this framework, implementation fidelity is measured by the extent to which the implementers adhere to the intervention as intended by the designers. Within the concept of adherence, content, frequency, coverage and duration are measured. The framework also describes potential moderating factors (moderators) which include the complexity of the intervention, facilitation strategies such as guidelines, monitoring and feedback, quality of delivery of the intervention and participant responsiveness that refers to enthusiasm and acceptance among those receiving and those delivering the intervention. In adapting the framework to study partograph use in the JSY programme, we studied two moderators-facilitation strategies and participant 
responsiveness, and coverage subcategory of adherence, having considered feasibility and data availability. The study framework adapted from Carroll et als framework for implementation fidelity is shown in figure 1 .

\section{Settings}

The study was conducted in the large, central Indian province of Madhya Pradesh (MP). Two-thirds of MP's 72 million population are rural $^{24}$ and one-third live below the poverty line. MP has poorer health indicators relative to Indian averages. Infant mortality stands at $67 /$ 1000 births, ${ }^{25}$ which is the highest in India, and maternal mortality ratio (MMR) is 277 maternal deaths/ 100000 births. $^{26}$ The public health sector is the dominant provider of obstetric services in the province; the private health sector is small, concentrated in urban areas and unaffordable for the majority. In MP, the JSY programme has functioned largely through public sector facilities. The public health system has a three tiered network of facilities: each district in the province has a tertiary level district hospital (DH) providing care for obstetric complications as well as normal birth to women either arriving directly or referred from community health centres (CHCs) that are secondary care facilities within districts. CHCs in turn cater to women arriving directly or referred from primary health centres (PHCs) in the periphery. All pregnant women in MP are eligible for JSY participation. The JSY provides a cash transfer of about US $\$ 31$ to rural mothers and US\$22 to urban mothers. During implementation of the JSY in MP, the institutional birth proportion has increased from $31 \%$ in 2007 to $72 \%$ in $2012,{ }^{15}$ benefiting seven million women so far. $^{27}$

\section{Study districts and facilities}

As an administrative unit within a province, each district has a population between 1 and 1.5 million. Of the 50 districts in MP, three heterogeneous districts were selected for this study based on their geographic location and differing socioeconomic level of development (as indicated by human development indices). Public facilities that reported at least 10 deliveries a month in the past 6 months were selected for the study.



Figure 1 Study framework adapted from conceptual framework for implementation fidelity. ${ }^{23}$ 


\section{Study design}

This was a mixed methods study using quantitative as well as qualitative methods. We performed (1) a record review of intrapartum case records to assess the extent of partograph use across a range of public facilities implementing the JSY programme, (2) a vignette-based survey to assess competence of staff providing care in the programme at using the partograph and (3) semistructured interviews with staff to explore the 'why' questions triggered from the quantitative findings.

The study was conducted between February 2012 and April 2013.

\section{Data collection}

Details of data collection additional to the overview in table 1 are described below.

\section{Record review}

Trained researchers selected the obstetric records from the delivery register in each facility. The first record was randomly selected from the last 1 year, while subsequent ones were selected using a sampling interval (calculated by dividing the total deliveries at the facility in the last 1 year by the desired sample size for the facility). The records were hand-written case sheets located in store rooms, as unorganised piles of papers or records bundled by month. Where a record was missing, it was replaced by the next record for the same date in the delivery register.

Each selected record was screened to identify if it contained a partograph. Our initial plan was to assess the partograph against a list of criteria. However, in our pilot study, conducted in a neighbouring district using a sample of 20 records, we observed that details such as time of admission, clinical condition on arrival and subsequent vaginal examination findings were generally not recorded, and this did not allow us to assess if the partograph was used appropriately. We therefore limited our study to documenting if any attempt to use a partograph was made and considered one as filled if at least one clinical finding was recorded on the graph that plots cervical dilation against time.

\section{Vignette-based survey}

The model for construction of case vignettes proposed by Heverly $e t a l^{28}$ was used to guide the vignette development process. Three vignettes were developed for this study: (1) normal progress of labour, (2) slow progress with signs of fetal distress and (3) non progress of labour. Each case description in the vignette provided clinical findings on cervical dilatation, frequency and duration of uterine contractions, pulse, blood pressure, temperature and fetal heart rate with time. Content validity of the vignettes and scoring method was established before the survey by expert opinion while face validity was assessed by discussion with delivery room nurses from all levels of facilities. The standard responses were developed based on the recommended practices for SBAs in India. ${ }^{29}$ The maximum possible score for a respondent was 10 . The vignettes and scoring system are in the online supplementary file 1 . A pilot test $(n=20)$ was conducted in a neighbouring district, not included in this study, to test feasibility of administering the vignette survey. All nurse-midwives who are routinely deployed in the delivery room or their supervisors in the selected facilities were invited to participate in the survey, as they conduct the majority of births in the JSY programme and are expected to monitor progress of labour. The interviewer explained that the survey aimed to assess the overall competence of nursing staff providing obstetric care in the JSY programme and was not an individual assessment. On average, respondents took $10 \mathrm{~min}$ to complete the survey while on the ward. The response sheet did not include names or personal identifiers.

Each respondent was presented with one vignette asking her to plot the findings on the simplified WHO partograph-which is routinely provided in public facilities. Following the plotting, respondents were asked to answer an open-ended question seeking decisions

\begin{tabular}{|c|c|c|c|}
\hline Method & Sample selection & Sample size & Objective \\
\hline $\begin{array}{l}1 \text { Obstetric case } \\
\text { record review }\end{array}$ & $\begin{array}{l}\text { Quota sampling: } 500 \text { records/district; } \\
\text { Facility quota proportionate to } \\
\text { delivery case load at the facility } \\
\text { based on deliveries in public facilities } \\
\text { in the district; } \\
\text { Systematic random sampling using } \\
\text { delivery registers at facilities }\end{array}$ & Total: 1500 & $\begin{array}{l}\text { To determine extent of partograph } \\
\text { use (adherence) }\end{array}$ \\
\hline 2 Vignette survey & $\begin{array}{l}\text { All staff (qualified birth attendants) } \\
\text { working as delivery room nurses at } \\
\text { study facilities }\end{array}$ & $\begin{array}{l}233 \text { (Nurse-midwives/ } \\
\text { Auxiliary } \\
\text { nurse-midwives) }\end{array}$ & $\begin{array}{l}\text { To assess competence of staff in } \\
\text { using partograph to monitor } \\
\text { progress of labour (ability) }\end{array}$ \\
\hline $\begin{array}{l}3 \text { Semistructured } \\
\text { interviews with } \\
\text { providers }\end{array}$ & $\begin{array}{l}\text { Purposive selection using maximum } \\
\text { variation sampling }\end{array}$ & $\begin{array}{l}11 \text { (Nurse-midwives and } \\
\text { obstetricians) }\end{array}$ & $\begin{array}{l}\text { To explore staff 'buy in' to policy } \\
\text { of routine partograph use } \\
\text { (responsiveness) }\end{array}$ \\
\hline
\end{tabular}


regarding care of the labouring woman presented in the vignette.

The first two authors independently marked the participants' responses against the standard responses.

\section{Semistructured interviews with providers}

Eleven interviews were conducted with nurse-midwives (9) and obstetricians (2) who work in labour rooms in a range of the study facilities of two districts. Length of experience and qualification were considered to ensure a range of responses. Using a topic guide, their experiences and perceptions of using the partograph, including any barriers to use and any training received, were explored. The lead author conducted the interviews in a location chosen by the respondent within the health facilities. They were conducted in the local language, were audiotaped with permission of the respondent and lasted for approximately $40 \mathrm{~min}$.

\section{Analysis}

Data from the record review were entered in REDCAP software and the scores from the vignette survey were entered into Excel spread sheets. STATA V.10 was used for analysis. Agreement between competence scores by the two raters was assessed using intra class correlation. Results are presented using descriptive statistics.

Audiotapes from the interviews were transcribed verbatim and then translated into English. Transcripts were read several times to develop a coding framework, which was finalised through consensus among coauthors. A thematic framework analysis approach was used. ${ }^{30}$ The coding framework was applied to all data, the coded data were segregated, compared across cases and contrasted with other codes to identify patterns and relations in the data, and develop explanations. The OPENCODE $^{31}$ software was used for data management.

\section{RESULTS}

\section{Characteristics of study facilities and participants}

The study districts had 73 facilities eligible for the study and all were included. Table 2 presents the distribution of the study facilities by districts and by levels of care, the number of case records reviewed and number of participants for the vignette survey at each level.

Participants: Of the 256 birth attendants who were eligible for the vignette survey, $91 \%$ participated. Only one potential participant refused, the other non-participants were away on training or on leave.

The birth attendants either hold an 18-month auxiliary nurse midwife (ANM) qualification, or have undergone 3-year training in general nursing and midwifery (GNM) or 4-year training to qualify with Bachelors of Science (BSc) in nursing. Two-thirds of ANMs in our study were at primary care facilities, though some were also posted at higher level facilities. GNMs and BSc nurses were mostly at secondary and tertiary care facilities. This distribution was similar across the three districts. The characteristics of participants are described in table 3 .

Among the participants for the semistructured interviews, both obstetricians were based at $\mathrm{DHs}$ and had more than 5 years' experience while of the nine nurses (including 2 ANMs) three were based at DHs and six were working at CHCs. Seven of the nurses had over 5 years' experience and six of them were working as head nurses.

\section{Adherence to partograph use-results from the record review}

The review of case records showed very low use of partographs. Of the 1466 records reviewed, 6\% records had a partograph with at least one assessment of cervical dilatation plotted against time. Most case records where a partograph was used were found at DHs, while none was found at the PHCs.

\section{Ability to use a partograph-competence scores from the vignette-based survey}

The reliability coefficient for inter rater reliability of scores assigned by two raters was 0.97 (95\% CI 0.95 to 0.99), indicating strong agreement.

The scores for competence in plotting a partograph ranged between 0 and 9 (of 10) with a mean score of 1.08 , which approximated to $11 \%$ of the maximum

\begin{tabular}{|c|c|c|c|c|c|c|}
\hline Facility level/districts & District 1 & District 2 & District 3 & Total facilities & $\begin{array}{l}\text { Number of } \\
\text { records } \\
\text { reviewed }\end{array}$ & $\begin{array}{l}\text { Number of vignette } \\
\text { survey participants } \\
\text { at each level of care }\end{array}$ \\
\hline Primary & 20 & 21 & 12 & 53 & 414 & 97 \\
\hline Secondary & 6 & 5 & 6 & 17 & 660 & 99 \\
\hline Tertiary & 1 & 1 & 1 & 3 & 392 & 37 \\
\hline Total & 27 & 27 & 19 & 73 & & \\
\hline Number of records reviewed & 513 & 497 & 456 & & $1466^{*}$ & \\
\hline Number of survey participants & 73 & 94 & 66 & & & 233 \\
\hline
\end{tabular}


Table 3 Characteristics of participants in the vignette survey $(n=233)$

\begin{tabular}{ll}
\hline Characteristic & $\begin{array}{l}\text { Median (range)/ } \\
\text { percentage }\end{array}$ \\
\hline Age & $36(21-65)$ years \\
Total experience & $10(0.5-40)$ years \\
Experience in obstetrics & $5(0-39)$ years \\
Average deliveries per month & $15(0-300)$ \\
Proportion SBA trained & $56 \%$ \\
Proportion women & $100 \%$ \\
Qualification & \\
$\quad$ Auxiliary nurse midwife (ANM) & $66 \%$ \\
$\quad$ General nurse and midwife (GNM) & $28 \%$ \\
$\quad$ Bachelor of science (nursing) & $6 \%$ \\
\hline
\end{tabular}

score. Figure 2 shows clustering of the scores to the lower end of the histogram. Of all participants, $75 \%$ scored below $15 \%$ of the maximum possible score and only $3.4 \%$ scored above $50 \%$ of it. Despite an inability to plot findings on the partograph, 27.5\% participants were able to arrive at a correct clinical decision regarding first line care for the condition presented.

\section{Responsiveness to partograph use-findings from interviews on staff 'buy in'}

Three themes emerged from the interviews: use partographs rarely and retrospectively; training does not support correct use of partographs; and partographs can be useful but are not feasible. These themes are illustrated in figure 3 and in the following sections, supported by direct quotations from the participants.

\section{Use partographs rarely and retrospectively}

Most staff reported that it was not possible to plot a partograph for each woman during labour. Many respondents reported an increased workload since the JSY began; there was a shortage of staff relative to workload and therefore time constraints. This high workload and time constraint precluded frequent and timely

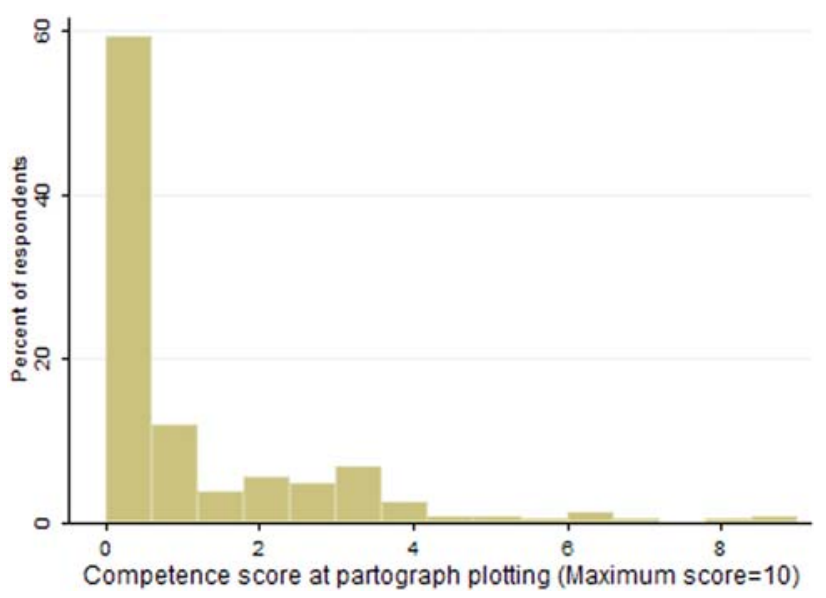

Figure 2 Histogram of scores for plotting a partograph. monitoring, and led to partographs being often filled incompletely, since the findings on maternal and fetal parameters that needed to be assessed were left blank.

I am mostly alone on duty and many women come for delivery nowadays, so how can I fill the partograph at that time. I fill it up later. (Head nurse)

We don't fill the main things (clinical parameters), we just fill the name, address on the first page and then whether it was a boy or a girl born; that's all. (Head nurse)

How could you think it is feasible in our kind of facilities with so much delivery load? (Obstetrician)

Respondents reported that they filled the partograph retrospectively at times, to ensure the record was complete. A head nurse pointed out that as a supervisor, she was aware this was not appropriate but indicated they were in a way compelled to do so since the use of partographs was monitored by authorities, and inspectors briefly looked through records to see if the partographs were filled. Others said that it was an unwritten norm to complete partographs when expecting such an inspection visit.

Actually it should be filled earlier, what we are doing is wrong but occasionally some director or someone will come and ask about it, we have to show them that we are filling it, so we are implementing it this way here. (Head nurse)

A head nurse claimed that she had instructed staff at her facility to use the partograph appropriately but they had refused.

...If I tell them to fill it during delivery, they reply we will fill it after the delivery. What can I do? (Head nurse)

Respondents described that at facilities where nurses conduct multiple consecutive deliveries, they forget findings before they can be entered in a partograph. This appeared more common at DHs where nurses switch between delivery tables to cater to many women simultaneously. In addition, respondents reported that some nurses continuously conducted deliveries while others wrote the case records including the partograph and they seldom had any communication about the clinical findings.

\footnotetext{
We are two sisters (nurses) here, so one fills the partograph and the various records and the other one conducts the deliveries. (Head nurse)
}

Few respondents reported irregular supply of printed partographs as a reason for not using the partograph routinely. 
Figure 3 Themes and their composition-finding from interviews with providers.

\begin{tabular}{|c|c|}
$\begin{array}{c}\text { Use partograph rarely } \\
\text { and retrospectively }\end{array}$ & $\begin{array}{c}\text { Training does not } \\
\text { support correct use of } \\
\text { partograph }\end{array}$ \\
$\begin{array}{l}\text { - No monitoring } \\
\text { hence no concurrent }\end{array}$ & $\begin{array}{c}\text { - Underconfidence/ } \\
\text { inexperience at }\end{array}$ \\
plotting & using partograph \\
- Plot it & Standard use not \\
retrospectively & demonstrated \\
- Staff shortage and & during pre-service \\
increased workload & and in-service \\
preclude plotting a & education, rather \\
partograph & include \\
& retrospective use \\
& \\
\hline
\end{tabular}

Partograph can be

useful but is not

feasible

- Useful as an aid for communication amongst staff

- More useful at facility level other than their own

- Reservations about feasibility at routine use - women arriving too close to delivery

\section{Training does not support correct use of the partograph}

The poor competence at using the partograph was found rooted in the poor training staff received. Respondents reported that they were not confident in using one after their training. Although it was mandatory for every student to complete 10 partographs as part of their practical training, staff recollected that these were generally filled post-facto after observing the delivery rather than during labour at the bedside.

We had a casebook with the graph, there were so many of us (students) so we could not do anything there (in the delivery room); we just watched; we used to fill it up later, after going to the hostel. (Head nurse)

I feel afraid that I might fill it incorrectly (and then someone could point it out), so I never fill it. (Auxiliary nurse)

A similar gap in training was reported about the in-service SBA training. The nurses recollected that as in-service trainees, they examined women and reported the findings to senior nurses, who then filled the partograph. One participant recollected a session on partograph use during the SBA training:

They had explained it in class but they didn't explain it at bed side. (Head nurse)

\section{Partographs can be useful but are not feasible}

The partograph was seen as a useful tool theoretically, but staff doubted its practical utility. It was seen as an aid to communication about progress of labour among staff in the facility and also between facilities in case of referral. With regard to referral it was seen to be useful for staff either at sending or at receiving facility level, responses invariably pointing to usefulness at facilities other than their own.

People at CHCs, PHCs and small hospitals should fill it because they have to refer women, but we have a doctor available 24 hours here (at $\mathrm{DH}$ ), we can call her and immediately show her the patient directly (so for us it is not needed as much as for them). (Head nurse)

It is good for high risk cases and those we have to refer from here (CHC). It is useful for the staff at DH (where the woman is referred). (Head nurse)

Many respondents reported that use of the partograph ensures important parameters such as blood pressure and fetal heart rate are examined in a timely manner. An obstetrician described an incident of a stillbirth where she felt that use of the partograph could have been lifesaving.

I saw the patient at $11.30 \mathrm{pm}$, she was $4 \mathrm{~cm}$ dilated with leaking membranes. Next morning I asked my duty doctor about her progress. She told me the same findings as in the night. I felt if the duty doctor had plotted her partograph she would have at least called me earlier. (Obstetrician)

A minority of respondents explained that the partograph could be useful as an aid for safety of staff in the event of a legal issue around care during labour and delivery.

Although staff did not deny the utility of the partograph as an aid in monitoring progress of labour, they felt this was only possible under ideal conditions. They had reservations as to the feasibility of its routine use in everyday settings, particularly because women generally arrive close to delivery, leaving no opportunity for the use of a partograph while staff shortages prevail.

If we have 10 women coming, 5 will come in full dilatation, so we can't fill the partograph. (Head nurse)

\section{DISCUSSION}

This study adds to the knowledge regarding implementation of the partograph in low-resource settings. It uses the implementation fidelity framework and a mixed methods approach to provide a deeper understanding into the current implementation scenario, and identifies 
bottlenecks to partograph use in the JSY programme for in-facility births in India. Application of the analytical framework for implementation fidelity enables generalisability of study results. The findings provide insights to consider when recommending for or against routine use of the partograph in the context of the cash transfer programme in India and in resource-constrained settings generally.

The interpretation of our results, in light of Carroll's framework, to study implementation fidelity, is presented in figure 4. By studying aspects of implementation such as adherence, abilities and responsiveness to partograph use the study finds a low implementation fidelity of partograph use. It identifies provider training, supervision, practice environment, including user compliance, as potential moderators of partograph use that influence adherence and hence the intended outcomes from partograph use. The results indicate high potential to improve quality of care in the JSY programme by improving the potential moderators to adherence that this study identifies.

The moderator 'practice environment' that this study identifies is in agreement with Hasson $e t a l^{22}$ who applied Carroll's framework to study complex health interventions and suggested 'context' as a potential moderator. Given that implementation fidelity is dependent on interrelationships between moderators, overall, our findings call for consideration of the context of partograph use in the JSY programme by taking into account the factors related to organisational culture that affect practice environment and hence the implementation.

\section{Partograph use in the JSY is low, but low use is not} unique to India

Our findings of low use of the partograph are consistent with another study from Southern India, ${ }^{33}$ which reported partograph was used at $3.8 \%$ births observed. Studies from other low income and high maternal

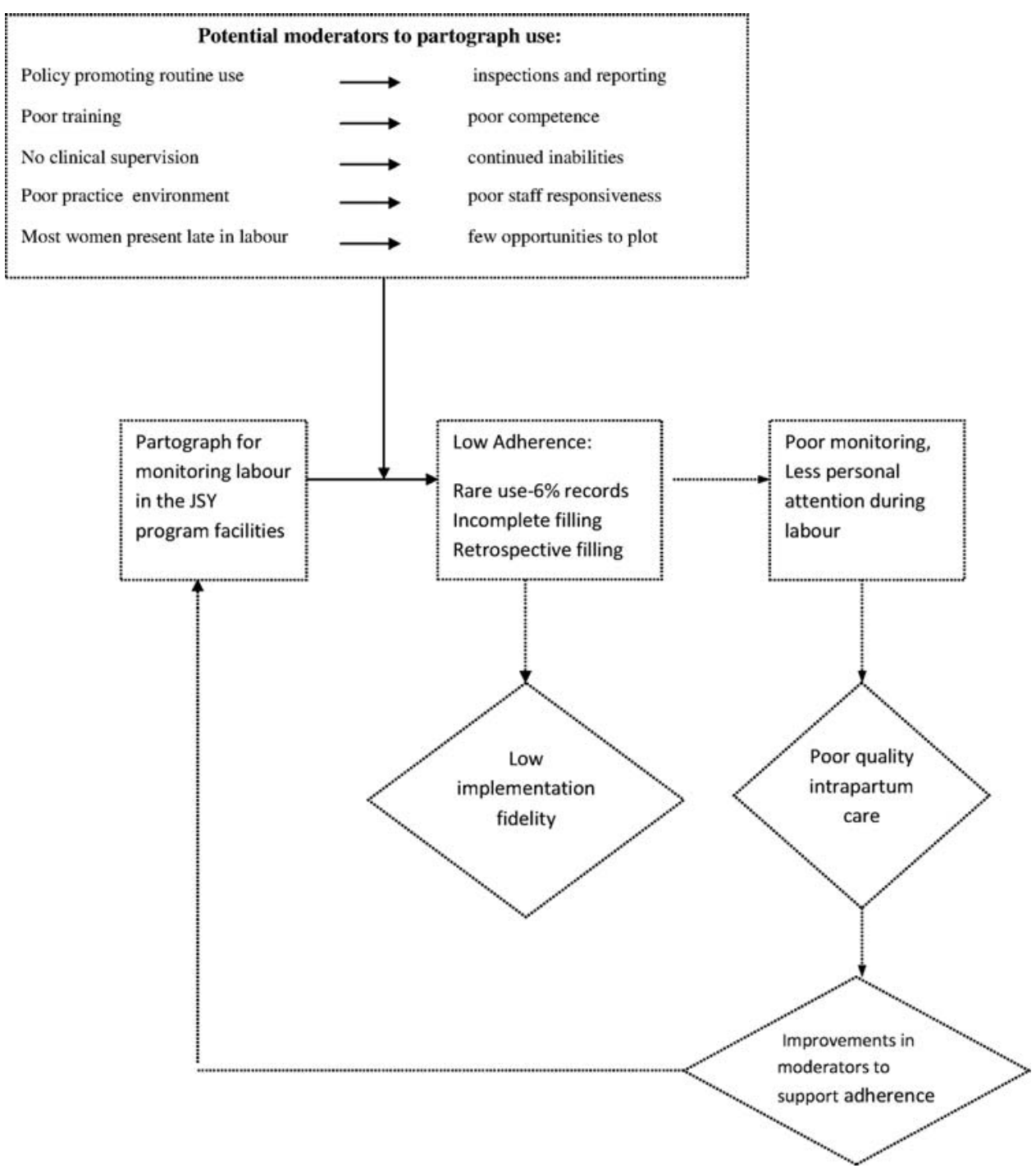

Figure 4 Implementation fidelity of partograph use in the Janani Suraksha Yojana (JSY) programme in Madhya Pradesh (MP), India-an application of Carroll's implementation fidelity framework. 
mortality countries such as Ethiopia, ${ }^{34-36}$ Ghana ${ }^{37}$ and Tanzania, ${ }^{38}$ have also reported low knowledge and utilisation of the partograph. Similar reasons surrounding training and poor 'buy-in' were reported by a study in Malawi, where only $3.9 \%$ of health workers could plot the partograph correctly. Our findings indicate problems with pre-service education as well as with in-service training leading to poor competence and non-use of the partograph. Given these inadequacies in training, it may be unrealistic to expect staff to be competent at using the partograph. Student midwives in Kenya experienced similar lack of hands on partograph training in the labour ward. ${ }^{39}$ The other two common reasons for not routinely using the partograph in our study were staff shortages and late arrival of women in labour. The issue of staff shortages is a long standing challenge in many low-and middle-income countries including India. ${ }^{40}$ Currently $33 \%$ of nursing staff posts at non-DHs in MP were vacant. ${ }^{41}$ Hulton et $a l,{ }^{42}$ in their framework to assess quality of institutional delivery care, consider late arrival of women as a sign of poor quality of care, indicating women delay going to facilities due to perceived discomfort and rude behaviour of staff.

\section{Partograph use requires better training and a conducive work environment}

Retrospective completion of partographs mainly for inspections, as seen in this study, is indicative of poor supervisory mechanisms. Such bureaucratic use of the partograph is also reported from Brazil. ${ }^{43}$ The partograph use found in this study, although low, was highest in the DHs where the availability of obstetricians may render it relatively less useful at suspecting abnormal progress of labour than at peripheral centres. It is unlikely that staff value completing a partograph when they do not use it to make decisions on interventions during labour. Popular use of partographs would require a clinical environment where decision-making is systematic and evidence based. This is not always the case in the study settings, where decision-making is rarely protocol based, as documented by our observational study of delivery care under the programme. ${ }^{44}$ Difficulties with understanding the application of the partograph in practice have been reported from other low-income settings as well and demand innovative ways of improving training in partograph use. ${ }^{45}$ Overall, the poor 'buy in' to partograph use that this study reports is not surprising. We would rather argue that the facility environment characterised by staff shortage, high work load and routines where monitoring of labour is not usually practiced (or feasible because of women coming very close to or in the second stage) does not encourage the use of a partograph among staff. It is important to note that previous studies $^{9} 4647$ that have reported an association between partograph use and positive labour outcomes were necessarily coupled with proper case management protocols, and show that partograph use needs continuous reinforcements and quality assurance. ${ }^{46}$
We therefore conclude that the structures, circumstances and work culture in the study facilities are not conducive to routine use of the partograph. Our findings add criticality of the practice environment to the commonly known challenges to routine use of partograph reported from other contexts, such as insufficient knowledge and workload pressure. ${ }^{11}$

\section{Routine partograph use as an indicator of quality of normal delivery care}

The JSY was launched in the context of a larger health system reform in India-the National Rural Health Mission. As part of the Mission's attempts to improve monitoring processes in the health system, facilities under the JSY were required to report the proportion of women in labour who were monitored using the partograph. We were unable to find literature or anecdotal evidence about how these data are being used. Our findings not only suggest a low use of the partograph but, importantly, reveal the realities concerning the way the partograph is currently used-in retrospect and more for bureaucratic reasons. Therefore, measuring the routine use of partographs in the current conditions would seem a poor choice of indicator of quality of normal delivery care. It is unlikely to support quality improvement, unless the conditions for routine use of the partograph are met and it is used for monitoring labour as intended. It is also important to consider that the majority of women arrive in the second stage; hence frequency of partograph use cannot be a useful indicator, as reported by other studies. ${ }^{48}$ Although there have been suggestions for the routine use of the partograph as a new indicator of quality of care for normal delivery, ${ }^{5}$ our findings suggest caution with using it in the current context.

\section{Partograph use in the JSY context: considerations and potential way forward}

India has a high maternal mortality and the share of deaths from obstructed labour remains steady at $5 \%{ }^{27} 49$ despite increased coverage of institutional births through the JSY, active promotion of partograph use and investments in providing emergency obstetric care. It is important to note that obstructed labour is often a precursor to maternal deaths from haemorrhage and sepsis, and that classification of causes of maternal mortality is based on the proximate causes of death. Hence although the leading causes of maternal deaths in India are haemorrhage $(38 \%)$ and sepsis $(11 \%),{ }^{49}$ routine and correct partograph use that can prevent deaths from obstructed labour, appears to be important to dent maternal mortality. Furthermore, routine partograph use could ensure personal attention to women and improve quality of routine care that can positively impact outcomes. For instance, it could prevent delays in recognising signs of obstetric complications and facilitate timely initiation of treatment or referral. It is important to consider that most births in the JSY 
programme are conducted by nurse midwives who have limited competence in midwifery, ${ }^{50}$ and care is not always under supervision of obstetricians. In such a context, routine use of the partograph could greatly improve care and prevent adverse outcomes. This would require building competence, providing supportive supervision, ensuring SBAs understand the importance of monitoring of labour, and creating a conducive environment by addressing staff shortages and ensuring women arrive at facilities in the first stage of labour. Findings from recent research by Underwood, ${ }^{51}$ demonstrating the use of digital pen technology to address training barriers to partograph use at a Kenyan hospital, offer innovative solutions that could be considered in other developing country contexts.

Limitations: First, although we report a low use of partographs, we were unable to conclude from the records if partograph use was recommended in all cases studied. There may be cases where actions other than partograph use, such as immediate referral or caesarean section, should be followed. Although a possibility, this number is likely to be small, thus not affecting the trend of our results. We were limited by the quality of clinical records and unable to determine the proportion of women who arrived late in labour. Second, our study is restricted to public facilities in MP and our findings may not be generalisable to public facilities in other provinces or to private facilities.

\section{CONCLUSIONS}

Study of implementation fidelity of an intervention can provide useful information to guide improvement in outcomes intended from the intervention. This study finds low utilisation of partographs for monitoring labour in the JSY programme and limited abilities of staff to use this tool in an environment where staff show poor buy-in to routine use of the partograph. Creating a policy to promote routine use of the partograph is not enough to ensure its implementation; other potential moderators to its adherence such as training, supervision, staff 'buy in' and user compliance need attention. Partograph use can result in improved quality of care and health outcomes in the JSY programme, only if the issues of work overload, staff shortages, training and supervision are addressed, so that staff finds a conducive work environment to use the partograph and women find it beneficial to present early in labour.

Acknowledgements The authors thank Dr Manish Singh for assistance during data collection. The authors thank Dr B Subha Sri, Dr Snehal Deshmukh, Dr P R Tekwani and Dr M B Swamy for technical advice. The authors are grateful to all study participants and to the MATIND study team for their cooperation. The authors acknowledge support from the National Rural Health Mission, Government of Madhya Pradesh.

Contributors SC, ADC and JR conceptualised and designed the study. SC and SU acquired and analysed the data. SC wrote the first draft. SU contributed to revising the draft. $A D C$ and JR participated in interpretation of results and revised the draft. All authors read and approved the final version.
Funding This research was funded by the EU FP7 grant to project MATIND. Competing interests None declared.

Ethics approval Approval to conduct this study was granted by the Institutional Ethics Committee of the R D Gardi Medical College, Ujjain, India.

Provenance and peer review Not commissioned; externally peer reviewed.

Data sharing statement No additional data are available.

Open Access This is an Open Access article distributed in accordance with the Creative Commons Attribution Non Commercial (CC BY-NC 4.0) license, which permits others to distribute, remix, adapt, build upon this work noncommercially, and license their derivative works on different terms, provided the original work is properly cited and the use is non-commercial. See: http:// creativecommons.org/licenses/by-nc/4.0/

\section{REFERENCES}

1. Graham WJ, McCaw-Binns A, Munjanja S. Translating coverage gains into health gains for all women and children: the quality care opportunity. PLoS Med 2013;10:e1001368.

2. Van den Broek NR, Graham WJ. Quality of care for maternal and newborn health: the neglected agenda. BJOG 2009;116(Suppl 1): 18-21.

3. Graham WJ, Campbell OM. Maternal health and the measurement trap. Soc Sci Med 1992;35:967-77.

4. World Health Organization. Monitoring emergency obstetric care: a handbook. Geneva: World Health Organization, 2009.

5. Gabrysch S, Civitelli G, Edmond KM, et al. New signal functions to measure the ability of health facilities to provide routine and emergency newborn care. PLoS Med 2012;9:e1001340.

6. World Health Organization. WHO partograph in management of labour. Lancet 1994;343:1399-404.

7. Lavender T, Hart A, Smyth RM. Effect of partogram use on outcomes for women in spontaneous labour at term. Cochrane Database Syst Rev 2013;(7):CD005461.

8. Fawole AO, Hunyinbo KI, Adekanle DA. Knowledge and Utilization of the Partograph among obstetric care givers in South West Nigeria. Afr J Reprod Health 2008;12:22-9.

9. Bosse $\mathrm{G}$, Massawe $\mathrm{S}$, Jahn A. The partograph in daily practice: it's quality that matters. Int J Gynecol Obstet 2002;77:243-4.

10. Lavender $\mathrm{T}$, Lugina $\mathrm{H}$, Smith $\mathrm{H}$. The partograph: a life-saving tool for African midwives. Trop Doct 2007;37:191-2.

11. Mathai M. The partograph for the prevention of obstructed labor. Clin Obstet Gynecol 2009;52:256-69.

12. Groeschel N, Glover P. The partograph: used daily but rarely questioned. Aust J Midwifery 2002;14:22-7.

13. Lavender T; Malcolmson L. Is the partogram a help or a hindrance? An exploratory study of midwives' views. Pract Midwife 1999;2:23-7.

14. World Health Organization. Trends in maternal mortality: 1990 to 2013. Estimates by WHO, UNICEF, UNFPA, the world bank, and the United Nations population division. Geneva: World Health Organization, 2014.

15. Registrar General of India. Maternal mortality ratio estimates, sample registration system 2010-12. New Delhi: Government of India, 2012.

16. Ministry of Health and Family Welfare. Janani SurakshaYojana: revised guidelines for implementation 2006. New Delhi: Government of India, 2006.

17. Ministry of Health and Family Welfare. Guidelines for antenatal care and skilled attendance at birth by ANMs and LHVs 2005. New Delhi: Government of India, 2005.

18. Lim SS, Dandona L, Hoisington JA, et al. India's Janani Suraksha Yojana, a conditional cash transfer programme to increase births in health facilities: an impact evaluation. Lancet 2010;375:2009-23.

19. Randive B, Diwan V, De Costa A. India's conditional cash transfer programme (the JSY) to promote institutional birth: is there an association between institutional birth proportion and maternal mortality? PLOS ONE 2013;8:e67452.

20. Sri BS, Naidu S, Khanna R. An investigation of maternal deaths following public protests in a tribal district of Madhya Pradesh, Central India. Reprod Health Matters 2012;(39):11-20.

21. Chaturvedi S, Randive B, Diwan V, et al. Quality of obstetric referral services in India's JSY cash transfer programme for institutional births: a study from Madhya Pradesh Province. PLoS ONE 2014;9: e96773.

22. Dusenbury L, Brannigan R, Falco $M$, et al. A review of research on fidelity of implementation: implications for drug abuse prevention in school settings. Health Educ Res 2003;18:237-56. 
23. Carroll C, Patterson M, Wood S, et al. A conceptual framework for implementation fidelity. Implement Sci 2007;2:1-9.

24. Registrar General of India. Census of India 2011. New Delhi: Government of India, 2012.

25. Registrar General of India. Sample registration System 2011. New Delhi: Government of India, 2011.

26. Registrar General of India. Annual Health Survey of India 2012. New Delhi: Government of India, 2012.

27. Ministry of Health and Family Welfare. Analysis of Health Management Information System data. New Delhi: National Health Systems Resource Centre, 2013-2014.

28. Heverly MA, Fitt DX, Newman FL. The construction of case vignettes for evaluating clinical judgement: an empirical model. Eval Program Plann 1984;7:45-55.

29. Ministry of Health and Family Welfare. Skilled birth attendance-a handbook for auxiliary nurse midwives/lady health visitors and staff nurses 2010. New Delhi: Government of India, 2010.

30. Ritchie J, Spencer L, O'Connor W. Carrying out qualitative analysis. In: Ritchie J, Lewis J, eds. Qualitative research practice: a guide for social science students and researchers. London: Sage Publications, 2003:219-62.

31. OPENCODE. http://www.phmed.umu.se/english/divisions/ epidemiology/research/open-code/ (accessed 5 Jul 2014).

32. Hasson $\mathrm{H}$. Systematic evaluation of implementation fidelity of complex interventions in health and social care. Implement Sci 2010;5:67.

33. Spector JM, Agarwal P, Kodkany B, et al. Improving quality of care for maternal and newborn health: prospective pilot study of the WHO safe childbirth checklist program. PLOS ONE 2012;7:e35151.

34. Yisma E, Dessalegn B, Astaktie A, et al. Knowledge and utilization of partograph among obstetric care givers in public health institutions of Addis Ababa, Ethiopia. BMC Pregnancy Childbirth 2013;13:17.

35. Abebe F, Birhanu D, Awoke W, et al. Assessment of knowledge and utilization of the partograph among health professionals in Amhara region, Ethiopia. Sci J Clin Med 2013;2:26-42.

36. Yisma E, Dessalegn B, Astatkie A, et al. Completion of the modified World Health Organization (WHO) partograph during labour in public health institutions of Addis Ababa, Ethiopia. Reprod Health 2013;10:23.

37. Gans-Lartey F, O'Brien BA, Gyekye FO, et al. The relationship between the use of the partograph and birth outcomes at Korle-Bu teaching hospital. Midwifery 2013;29:461-7.

38. Duysburgh $\mathrm{E}$, Zhang $\mathrm{WH}, \mathrm{Ye} \mathrm{M}$, et al. Quality of antenatal and childbirth care in selected rural health facilities in Burkina Faso,
Ghana and Tanzania: similar finding. Trop Med Int Health 2013:18:534-47.

39. Lavender T, Omoni G, Lee K, et al. Students' experiences of using the partograph in Kenyan labour wards. Afr J Midwifery Womens Health 2011:5:117-22.

40. Rao M, Rao KD, Kumar AK, et al. Human resources for health in India. Lancet 2011;377:587-98.

41. Rural Health Statistics. https://nrhm-mis.nic.in/Ul/RHS/RHS\% 202011/RHS\%20-March\%202011-\%20Tables-\%20Final\%209.4. 2012.pdf (accessed 18 Feb 2014)

42. Hulton L, Matthews Z, Stones RW. Applying a framework for assessing the quality of maternal health services in urban India. Soc Sci Med 2007;64:2083-95.

43. AzevedoAguiar C, Gonçalves R, et al. Use of the partogram in labor: analysis of its application in different care models. Open J Obstet Gynecol 2013;3:1.

44. Chaturvedi S, De Costa A, Raven J. Increased institutional birth coveragedoes not mean improved skilled birth attendance: Study of intra-partum care in JSY cash transfer program in Madhya Pradesh, India. (Abstract) Third Global Symposium in Health Systems Research; Cape Town, October 2014.

45. Lavender T, Dame G, Omoni K, et al. A pilot quasi-experimental study to determine the feasibility of implementing a partograph e-learning tool for student midwife training in Nairobi. Midwifery 2013;29:876-84.

46. Fahdhy M, Chongsuvivatwong V. Evaluation of World Health Organization partograph implementation by midwives for maternity home birth in Medan, Indonesia. Midwifery 2005;21:301-10.

47. Fawole $\mathrm{AO}$, Fadare $\mathrm{O}$. Audit of use of the partograph at the University College Hospital, Ibadan. Afr J Med Sci 2007;36:273-8.

48. Kwast B, Poovan P, Vera E, et al. The modified WHO partograph: do we need a latent phase? Afr J Midwifery Womens Health 2008;2:143-8.

49. Registrar General of India. Special Bulletin on maternal mortality in India 2007-09. New Delhi: Government of India, 2011.

50. Chaturvedi S, Upadhyay S, De Costa A. Competence of nurse birth attendants in Indias's JSY conditional cash transfer program to promote institutional births: an assessment using case vignettes in Madhya Pradesh province. BMC Pregnancy Child Birth 2014;14:174.

51. Underwood $\mathrm{H}$. The Partopen: Using digital pen technology to improve maternal labour monitoring in the developing world [PhD Dissertation]. University of Colorado at Boulder, 2013. 